\title{
Dynamic Spectrum Access for Primary Operators: Exploiting LTE-A Carrier Aggregation
}

\author{
Khaled Qorany Abdel Fadeel \\ The 4G++ Project \\ Cairo University \\ Giza, Egypt, 12613 \\ kqorany@4gpp-project.net
}

\author{
Khaled Elsayed, Ahmed Khattab \\ Department of Electronics and \\ Communications Engineering, Cairo \\ University, \\ Giza, Egypt, 12613 \\ \{khaled,akhattab\}@ieee.org
}

\author{
Fadel Digham \\ National Telecommunications Regulatory \\ Authority, Ministry of Communication and \\ Information Technology \\ Giza, Egypt \\ fdigham@ntra.gov.eg
}

\begin{abstract}
In this paper, we address dynamic spectrum access for Primary Operators (POs) for LTE-Advanced systems. We propose a dynamic spectrum access framework that exploits the capabilities of carrier aggregation to efficiently utilize the unutilized spectrum which varies with time and space when static spectrum access policies are adopted. A spectrum owner (SO) adopts an auction scheme for accessing spectrum for the dynamic requests. Furthermore, we introduce an accurate model driven by real-life statics and LTE-A standard specifications for estimating the bandwidth required to satisfy the traffic demands of an operator's subscribers for a projected demand model. For the auction process, we map the spectrum access problem into a bounded knapsack problem which is solved using dynamic programming in pseudo-polynomial time. The solution uses the generalized second price strategy. Simulation results show more than $20 \%$ reduction in the required spectrum and up to $80 \%$ reduction in the average unutilized spectrum as compared with traditional static spectrum access due to the on-demand spectrum assignment.
\end{abstract}

Keywords- Dynamic spectrum access; auction; carrier aggregation; Bounded knapsack problem.

\section{INTRODUCTION}

Static long-term Spectrum Access (SSA) currently adopted by spectrum regulation authorities' results in an inefficient utilization of such a scarce resource: the wireless spectrum. Thus motivated, regulatory organizations are currently considering the adoption of dynamic spectrum access approaches that allow the different wireless operators to share the available spectrum based on their demands.

Such dynamic access policies would be required to take the temporal and geographical variations in the demands of different operators into account when allocating the spectrum shares of the individual operators to achieve the maximum allocation efficiency. In order to maximize the spectrum owner revenue and the users' social welfare, such dynamic spectrum access approaches typically adopt an auction or auction-like process.

In this paper, we present a dynamic spectrum access framework which exploits the capabilities of LTE-Advanced carrier aggregation. The main features of carrier aggregation (CA) are its backward compatibility with release 8 and 9 capabilities and the ability of spectrum aggregation of individual component carrier (CC) dispersed within and across different bands (intra/inter-bands). CA also allows the combination of CCs having different bandwidths. Hence carrier aggregation is considered a practical solution for the LTE spectrum fragmentation. These features offer significant flexibility for efficient spectrum utilization.

The proposed Dynamic Spectrum Access framework with Carrier Aggregation (DSA-CA) considers the bounded knapsack problem as an auction spectrum problem to satisfy the POs demand while maximizing the SO's revenue. We use system-level simulation unlike related work [1-10] to shows the main performance improvement effects achieved by the proposed DSA-CA framework with respect to conventional static spectrum access. Our results show more than $20 \%$ reduction in the required spectrum and up to more than $80 \%$ reduction in the average unutilized spectrum.

The rest of this paper is organized as follows: In Section II, the related research is summarized. We describe the system model in Section III. In Section IV, we propose the DSA-CA framework. In Section V, we describe the estimation of spectrum demand model in an LTE-Advanced environment. Simulation results are given in Section VI. Finally, Section VII concludes this paper.

\section{RELATED WORK}

The basic dynamic spectrum access model for primary operators was presented in [1] and [2] in which a primary user, i.e. the spectrum owner, rents spectrum to the secondary users. The model can be mapped to the service providers offering service to users directly. To address this model, the authors in [1] presented a hierarchical auction which comprises of three tiers: the spectrum owner tier, the primary operators tier, and the mobile users tier whereas the authors in [2] presented the flexible auction. In [3], the authors seek to exploit the variation in the loads of various radio-access networks to allocate the spectrum efficiently by dynamic spectrum scheme, where the spectrum owner performs Vickery auction periodically between service providers. Meanwhile, the model in [4] proposed a dynamic spectrum allocation process, where multiple wireless service providers compete to acquire the necessary spectrum band from a common pool of spectrum by different auction mechanisms. The model in [5] proposed a real-time spectrum auction to support spectrum allocation and pricing of a large number of secondary users under interference constrains whereas the work in [6] addressed the fairness issue when the secondary users suffer from interference constrains. Similarly, the models in [7] and [8] presented frameworks for dynamic allocation based on auction that is mapped into $0-1$

\footnotetext{
* This work is part of the $4 \mathrm{G}++$ project supported by the National Telecom Regulatory Authority of Egypt.
} 
knapsack problem and game theory based schemes that capture the interaction among the spectrum owner, primary service providers (PSPs) and end-users in a multi-provider setting.

On the other hand, only [9] and [10] consider DSA on top of carrier aggregation, however, they do not address the primary operator spectrum allocation problem (i.e., they assume each primary operator has already been assigned its spectrum share).

In this work, we target a similar DSA objectives as [1], [7] and [8]. However, our work distinguishes itself as 1) it is the first work to consider a realistic LTE system model to estimate the required spectrum shares of operators, 2) it maps the auction problem into a bounded knapsack problem given the realistic system assumption and LTE-A carrier aggregation constraints unlike [7] and [8] that consider a simplified 0-1 knapsack problem formulation, and 3) it presents an accurate system-level simulation results rather than the theoretical concept validations presented in all of the aforementioned works.

\section{SYSTEM MODEL}

We consider a mobile cellular environment that consists of $L$ Primary Operators (POs) in an LTE-Advanced (abbreviated LTE-A in the sequel) system and one Spectrum Owner (SO) which is responsible for spectrum management, e.g. the Federal Communications Commission (FCC) in USA and the National Telecom Regulatory Authority (NTRA) in Egypt.

We assume that the SO divides the whole service area, e.g. country, state, governorate, into smaller non-overlapping regions $J=\{1, \ldots, r\}$.

We assume that the SO divides the available spectrum into $W$ equal and homogeneous spectrum chunks which, for the sake of practicality, are defined to be compliant with LTE-A component carriers (CCs) definition.

The spectrum owner receives the spectrum requests periodically for a pre-specific lease time, decided and declared by the SO, from the POs in a sealed bid manner and then determines the winners set and the price to be paid by the winners. The objectives of the SO are to maximize its revenues and to increase the spectrum utilization as well as achieving social welfare. On the other hand, the objective of the POs is to maximize their benefits by reducing expenditures compared to the case of static allocation based on the projected maximum demand in a forecasting period. Another goal would be to allow the PO to attract more customers by offering attractive pricing for the periods of the day that the PO is allocated ample bandwidth.

\section{PROPOSED DSA-CA FRAMEWORK}

In this paper, we present a DSA framework that is applicable in practice using carrier aggregation capabilities of LTE-A. By making DSA dependent on CCs in a CA-based LTE-A, we achieve dynamic reaction to the cell load. This can be realized by a network management system (NMS) and proper signaling between the SO and the PO's by sending management messages that contain the list of allocated CCs in a given region. The PO's NMS will then activate the list of allocated CC's in its eNBs and at the end of predetermined lease time units deactivate them. The activation/deactivation mechanism is based on a combination of radio resource control
(RRC) messages and deactivation timers and takes a few time slots [11]. For the time being, issues like handling on-going connections at the end of a lease time are left for future work.

Due to the dynamic nature of the spectrum requests and the available supply, our framework uses sealed bid auction scheme when the sum of spectrum requests in a region exceeded the available supply. Auction schemes have proven successful in selling the scarce resources, and as such, are effective in selling spectrum resources when they are scarce. However, it is not suitable in the case of lack of competition between buyers due to abundance of supply. The spectrum owner is free to use any other pricing scheme when the sum of spectrum requests is less than the available supply, which is behind the scope of our work. The SO will broadcast the winning price(s) and corresponding assignments after the end of the auction.

\section{A. Auction Spectrum Access}

We consider a multi-unit auction wherein each PO submits its need of CCs for the predetermined time units as one request, i.e. bid in the language of auction theory, in each region.

POs do not have a hard requirement on their bids, i.e. POs prefer to obtain allocated spectrum from SO that is less than its need rather than getting nothing.

The problem described here is similar to the classical bounded knapsack problem [12], where the aim is to fill a sack of specific capacity with several identical items such that the total valuation of the items in the sack is maximized. Here, the sack represents the finite spectrum capacity the SO is willing to lease to the POs in such a manner that the revenue generated from these POs is maximized. In this regard, we propose a formulation based on the "Bounded Knapsack Auction" (BKA) problem.

We denote a strategy adopted by POs by a tuple $q_{i j}=$ $\left(f_{i j}, p_{i j}\right)$, where $f_{i j}$ denotes the number of CCs required by $\mathrm{PO} i$ in region $j$ and $p_{i j}$ denotes the price per $\mathrm{CC}$ that PO $i$ is willing to pay in region $j$.

We formulate the BKA as follows.

$$
\begin{gathered}
\max \sum_{i}^{L} p_{i j} x_{i j}, \forall j \in J \\
\text { Subject to } \\
\sum_{i}^{L} x_{i j} \leq W, \forall j \in J \\
0 \leq x_{i j} \leq f_{i j}, \forall i \in L, \forall j \in J \\
x_{i j} \text { is integer }, \forall i \in L, \forall j \in J
\end{gathered}
$$

where $x_{i j}$ denotes the number of CCs to be allocated to PO $i$ in region $j$. Our aim is to solve this problem to determine the set of winners in such a way to maximize revenue of spectrum owner which also improve spectrum utilization by allocating spectrum to POs who value it the most. Then, the SO determines the payments to be paid by the winners. In this paper, we consider the generalized second price bidding strategy. In second price auction, each bidder does not need to guess other bids but can offer a bid request which reflects his own valuation of the commodity (in our case the $\mathrm{CC}$ ), thus second price auction is considered an incentive-compatible auction [13]. The payment is set where the first winner pays the bid price of the second winner and the second winner pays the bid price of the third winner and so on except the last winner who pays his bid price. 
The bounded knapsack solution is standard. The simplest idea of solution is based on converting the bounded knapsack into 0-1 knapsack and then solving the 0-1 knapsack problem which is solved using the dynamic programming concept, i.e. breaking the problem into sub-problems that are solved by working backwards from the last stage. The bounded knapsack auction is solved in pseudo-polynomial time. We omit the details of the solution due to space considerations. The details of solution algorithm and its complexity analysis are available at [14] for interested readers.

To solve the bounded knapsack problem in (A), two input parameters are needed, namely, the number of CCs $f_{i j}$ and the corresponding bidding price $p_{i j}$. The following section presents a methodological approach for estimating the spectrum demand in an LTE-advanced environment. But the details of spectrum bidding price are outside the scope of this paper; we follow uniform distributions as will be shown in Section VI to mimic the bidding price behavior of operators. Our main target is to provide a system level framework for quantifying the potential utilization and economic gains of the DSA-CA based scheme which can further be improved using, per instance, optimized and sophisticated pricing/bidding models.

\section{ESTIMATION OF SPECTRUM DEMAND IN LTE-ADVANCED ENVIRONMENTS}

In this section, we present a methodological approach for accurately estimating the spectrum demand of POs on a certain time of a day in a specific region which serves as an input to our performance evaluation study. In order to estimate the spectrum demand of POs on a certain time of day in all regions, we present a detailed model for mapping the cells' traffic demand, taking into account the various LTE-A overheads, to the respective bandwidth. A preliminary version of this model was presented in [15].

\section{A. Traffic Model}

The cells' traffic demand varies dynamically in time and space. This dynamicity varies in accordance with the users' density distribution for each PO. The density distribution varies from region to region for the same PO. However, the user population is not active all the time and the users' activity differs from one PO to another and differs in space and time with the same PO. Our traffic model is driven by the set of statistical models and real-life measurements of LTE users' traffic in Europe reported in [16]. Unlike [16], our model explicitly estimates the generated cells' traffic demand of all POs. The following quantities are used in our model:

- The population densities $p_{d}\left[\right.$ citizen $\left./ \mathrm{Km}^{2}\right]$ in different deployments $d$.

- The mobile subscribers' percentage $m_{d}$ out of the total population in different deployment $d$.

- The broadband users' percentage, i.e. LTE and LTE-A users, $b_{d}=\sum_{k} s_{k}$ and the broadband active users' percentage $a_{d}$ in different deployment $d$, where $s_{k}$ denotes the fraction of broadband subscriber of the whole broadband subscribers for terminal $k$.

- The estimated rate $r_{k}$ required for each terminal $k$, e.g. Table I.
- The normalized daily traffic variations curve $\alpha(t)$.

- The Inter-Site Distance (ISD) of each cell.

\section{B. Traffic Mixes}

The estimated rate of each broadband user arises from different services, i.e. traffic mixes of a user. We assume that this traffic mix is the same for all users and consists of TCP services, e.g. downloading, browsing and gaming, and VoIP with different codecs, e.g. $12.65 \mathrm{kbps}$ and $6.06 \mathrm{kbps}$. The percentage of TCP and VoIP services are $80 \%$ and $20 \%$ respectively. Table II shows the percentage of each service and the average size of the application payload.

\section{LTE-A Overheads}

To be able to accurately transform the application/terminal bit rate into bandwidth, we need to account for the different forms of overheads added by the protocol stack comprised of TCP/IP/LTE layers and the specific structure of the LTE-A PHY later. The necessary overheads of LTE-A system is the summation of five types of overheads: multiple protocol layers overheads, the spectrum emission guard, reference signals overhead, control channels overhead and finally the synchronization signals overhead [17].

Protocol Overheads: Protocol overheads depend on the type of the service. In case of TCP services, the different protocol layers add 18 bytes to the application layer payload and in case of VoIP services, the different LTE layers adds 9 bytes to application layer payload after header compression [18]. Table II shows the percentage of the overhead at different services. The last column of table I provides the rates of different terminals after adding the protocol overheads. The other four overheads depend on the cell bandwidth.

Emission Guard Overhead: The emission guard overhead is the difference between the $\mathrm{CC}$ bandwidth and the bandwidth of the number of resource block in this channel, which equals $6,15,25,50,75$ and 100 resource blocks of $1.4,3,5,10,15$, $20 \mathrm{MHz}$ respectively, where the resource block consist of 12 subcarriers with a total bandwidth of $180 \mathrm{kHz}$ [17]. Thus the emission guard almost equals $10 \%$ of the CC size.

Reference Signals Overhead: The reference signals overhead differs based on the MIMO configuration. For $2 * 2$ MIMO, the reference signals occupy eight resource elements per resource block [17] which represents $9.5 \%$ of the CC size after excluding the emission guard.

Control Channel Overhead: The maximum control channel overhead occupies three OFDM symbols per subframe [17] which represents $21.4 \%$ of the $\mathrm{CC}$ size after excluding the emission guard.

Synchronization Overhead: The synchronization signals overhead occupy two resource elements for primary synchronization signals and another two resource elements for secondary synchronization signals per frame [17] which represents $0.23 \%$ of the $\mathrm{CC}$ size after excluding the emission guard. Table III reflects the effective residual spectrum after excluding the overheads, e.g. the effective spectrum of a 3 $\mathrm{MHz}$ bandwidth is equal to $[3-(3 * 10 \%)]-[(3-(3 *$ $10 \%))(9.5 \%+21.4 \%+0.23 \%)]=1.859 \mathrm{MHz}$. 
TABLE I. TERMINALS AND THEIR ESTIMATED RATES [16].

\begin{tabular}{|c|c|c|c|}
\hline Terminal $\boldsymbol{k}$ & $\begin{array}{c}\text { Mixes } \\
\boldsymbol{s}_{\boldsymbol{k}}[\mathbf{\%}]\end{array}$ & $\begin{array}{c}\text { Rate } \boldsymbol{r}_{\boldsymbol{k}} \\
{[\mathbf{M b p s}]}\end{array}$ & $\begin{array}{c}\text { Rate After Adding } \\
\text { Overhead } \boldsymbol{\mathcal { R }}_{\boldsymbol{k}}[\mathbf{M b p s}]\end{array}$ \\
\hline Heavy PC Users & 10 & 2 & 2.3698 \\
\hline Average PC Users & 10 & 1 & 1.1849 \\
\hline Average Smart Phone Users & 25 & 0.5 & 0.5924 \\
\hline Average Smart Phone Users & 25 & 0.25 & 0.2962 \\
\hline Average Tablet Users & 2.5 & 0.25 & 0.2962 \\
\hline Average Tablet Users & 2.5 & 0.125 & 0.1481 \\
\hline
\end{tabular}

\begin{tabular}{|c|c|c|c|c|c|}
\hline \multirow[b]{3}{*}{$\begin{array}{c}\text { Users' Traffic } \\
\text { Mixes }\end{array}$} & \multirow{2}{*}{\multicolumn{3}{|c|}{$80 \%$ TCP }} & \multirow{2}{*}{\multicolumn{2}{|c|}{$20 \%$ VoIP }} \\
\hline & & & & & \\
\hline & $\begin{array}{c}30 \% \\
\text { Down- } \\
\text { loading }\end{array}$ & $\begin{array}{l}20 \% \\
\text { Gaming }\end{array}$ & $\begin{array}{c}50 \% \\
\text { Browsing }\end{array}$ & $\begin{array}{c}50 \% \text { with } \\
\text { Codec } \\
12.65 \mathrm{kbps}\end{array}$ & $\begin{array}{c}\text { 50\%with } \\
\text { Codec } \\
6.60 k b p s\end{array}$ \\
\hline $\begin{array}{l}\text { App. Payload } \\
\text { [Bytes] }\end{array}$ & 1500 & 500 & 75 & 33 & 17 \\
\hline Overhead [\%] & 1.2 & 3.6 & 24 & 27.27 & 53 \\
\hline
\end{tabular}

TABLE III. EFFECTIVE SPECTRUM AfTER EXCLUdING ALL OverheAdS AT

\begin{tabular}{|c|c|c|c|c|c|c|}
\hline DIFFENT & $\mathbf{1 0}$ & $\mathbf{1 5}$ & $\mathbf{2 0}$ \\
\hline $\begin{array}{c}\text { CC Bandwidth [MHz] } \\
\text { Effective Spectrum } \\
\text { [MHz] }\end{array}$ & 0.743 & 1.859 & 3.099 & 6.198 & 9.297 & 12.396 \\
\hline
\end{tabular}

For a given inter site distance (ISD), the hexagonal cell area is given by $A=\frac{\sqrt{3}}{2} I S D^{2}$. Thus, the users' traffic demand curve of a $\mathrm{PO}$ over a cell in a deployment $d$ is determined by

$$
R_{d}(t)=\left(\frac{A_{d}}{1000}\right)^{2} p_{d} m_{d} a_{d} \alpha(t) \sum_{k} \mathcal{R}_{k} s_{k}[\mathrm{bits} / \mathrm{s} / \mathrm{cell}]
$$

At a specific average SINR and specific transmission mode we obtain the CQI from table III in [19] and, in turn, we compute the spectral efficiency from table 7.2.3-1in [20]. The final step is to divide the generated cell traffic demand curve (B) by spectral efficiency. The number of required CCs in this cell is obtained by dividing the cell spectrum demand by the respective number in of the last row of table III for the chosen $\mathrm{CC}$ allocation granularity.

\section{Performance Evaluation}

To demonstrate the effectiveness of the proposed DSA-CA framework, we conducted system level simulation experiments based on the above realistic LTE-A model. We consider a cellular network composed of three POs, i.e. $L=3$, which we call $\mathrm{A}, \mathrm{B}$ and $\mathrm{C}$. The SO divides the overall service area into two regions, i.e. $r=2$, which we refer to as $\mathrm{R} 1$ and $\mathrm{R} 2$. We consider that R1 region is composed of three LTE-A cells whereas R2 is composed of four LTE-A cells. We also assume that each PO has $5 \mathrm{MHz}$ that was statically allocated in all regions. The cells of the two regions are deployed as follows: the first region's cells and the first cell of the second region belong to dense urban deployment whereas the other three cells of the second region belong to urban deployment. Table IV shows the other simulation parameters of a specific day. This deployment is set to give a clear illustration of the gain resulting from the division of the service area into smaller regions to take advantage of the variations of spectrum demand between regions.

We assume that all broadband users of all POs have the same traffic mix as shown in table II, and then we assume that the average SNR at all cells is $3 \mathrm{~dB}$ which translates to spectral efficiency of $1.1758 \mathrm{~b} / \mathrm{s} / \mathrm{Hz}$ at $2 * 2$ MIMO configurations. We also assume that the operators reuse the winning CCs in the same region with a reuse factor of 1 .

Two particular cases of the daily traffic variations were identified, labeled scenario 1 and 2. Scenario 1 represents the case where all operators have identical or semi-identical traffic variation with time-of-day. Scenario 2 where there are shifts in the traffic demand pattern over the time-of-day.

There are some factors that affect the performance of DSA$\mathrm{CA}$, e.g. the available $\mathrm{CC}$ sizes in the market, the predetermined leasing time units and the message overheads between POs and SO. In Fig. 1 and Fig. 2, we show these effects based on the two key performance indicators maximum spectrum needed to serve all demands from the different cells and average unutilized spectrum, i.e. the average of the unused spectrum by all POs per hour [Hz/Hr], due to the difference of demands between cells. The message overheads will decrease whenever the DSA-CA increases pre-specified lease time units. Fig. 1 shows the huge gain of DSA-CA, almost in all cases of DSA-CA, which is up to $80 \%$ reduction in the unutilized spectrum in the case of CC $=1.4 \mathrm{MHz}$ and the DSA-CA is held every hour over conventional Static Spectrum allocation (SSA) which means that the operators got a maximum spectrum required all the time. This huge gain comes from the dynamic reaction to the operators load due to DSA-CA as compared to conventional SSA. Fig. 2 shows the spectrum which should be provided by the SO to cover all demands of POs. Also, Fig. 2 shows that at scenario 1 , the required spectrum quantity is close to the case of conventional SSA since the three operates have identical traffic variations with time-of-day. Meanwhile, the gain of DSA-CA become more significant (more than $20 \%$ reduction in the required spectrum) in scenario 2 in case of $\mathrm{CC}=1.4 \mathrm{MHz}$ and the DSA-CA is held every hour as compared to conventional SSA due to the time shifts in the traffic demand pattern over the time-of-day.

Fig. 3 gains insight about the revenue of SO, we assume all the spectrum bands are of equal value to all the POs. Note that through this simulation model, we use the notation unit instead of any particular currency. Firstly, the parameters of the auction, the reserve price of one $\mathrm{CC}$ for each $\mathrm{PO}$ is assumed to follow a uniform distribution with minimum 15, 40, 60 and maximum as $30,55,75$ units in the case of $\mathrm{CC}$ is equal to 1.4 $\mathrm{MHz}, 3 \mathrm{MHz}$ and $5 \mathrm{MHz}$ respectively. Secondly, the selling price follows a uniform distribution with minimum 15, 40, 60 and maximum 20, 45, 65 in case of CC equal $1.4 \mathrm{MHz}, 3 \mathrm{MHz}$ and $5 \mathrm{MHz}$ respectively when the sum of spectrum is less than the available supply. Furthermore, we assume that the bidders use auction histories of previous rounds to submit their bids in future rounds. Thus a winning bidder (a one which has all its needs or all the market in one DSA period) will try to submit a lower bid in the next DSA period in order to increase his surplus profit whereas a losing bidder will tend to increase his bid. Details of this price adjustment are outside the scope of this paper. 
TABLE IV. SimULATION PARAMETERS

\begin{tabular}{|c|c|c|c|c|c|c|c|c|c|c|c|c|c|c|c|c|c|c|c|c|c|}
\hline Regions & \multicolumn{9}{|c|}{ R1 } & \multicolumn{12}{|c|}{$\mathbf{R 2}$} \\
\hline Cell ID & \multicolumn{3}{|c|}{$\# 1$} & \multicolumn{3}{|c|}{$\# 2$} & \multicolumn{3}{|c|}{$\# 3$} & \multicolumn{3}{|c|}{$\# 1$} & \multicolumn{3}{|c|}{$\# 2$} & \multicolumn{3}{|c|}{$\# 3$} & \multicolumn{3}{|c|}{$\# 4$} \\
\hline$p_{d}\left[\right.$ citizen $\left./ \mathbf{K m}^{2}\right]$ & \multicolumn{3}{|c|}{3000} & \multicolumn{3}{|c|}{3000} & \multicolumn{3}{|c|}{3000} & \multicolumn{3}{|c|}{3000} & \multicolumn{3}{|c|}{1500} & \multicolumn{3}{|c|}{1500} & \multicolumn{3}{|c|}{1500} \\
\hline ISD [m] & \multicolumn{3}{|c|}{500} & \multicolumn{3}{|c|}{500} & \multicolumn{3}{|c|}{500} & \multicolumn{3}{|c|}{500} & \multicolumn{3}{|c|}{1000} & \multicolumn{3}{|c|}{1000} & \multicolumn{3}{|c|}{1000} \\
\hline POs & $\mathbf{A}$ & B & $\mathrm{C}$ & $\mathbf{A}$ & B & $\mathrm{C}$ & $\mathbf{A}$ & B & $\mathbf{C}$ & $\mathbf{A}$ & B & $\mathbf{C}$ & $\mathbf{A}$ & B & $\mathrm{C}$ & $\mathbf{A}$ & B & $\mathbf{C}$ & $\mathbf{A}$ & B & $\mathbf{C}$ \\
\hline$m_{d}[\%]$ & 35 & 25 & 20 & 40 & 20 & 20 & 35 & 20 & 25 & 20 & 30 & 35 & 20 & 25 & 40 & 25 & 25 & 35 & 20 & 30 & 35 \\
\hline$b_{d}[\%]$ & 75 & 75 & 75 & 75 & 75 & 75 & 75 & 75 & 75 & 75 & 75 & 75 & 50 & 50 & 50 & 50 & 50 & 50 & 50 & 50 & 50 \\
\hline$a_{d}[\%]$ & 16 & 16 & 16 & 16 & 16 & 16 & 16 & 16 & 16 & 10 & 10 & 10 & 10 & 10 & 10 & 10 & 10 & 10 & 10 & 10 & 10 \\
\hline
\end{tabular}

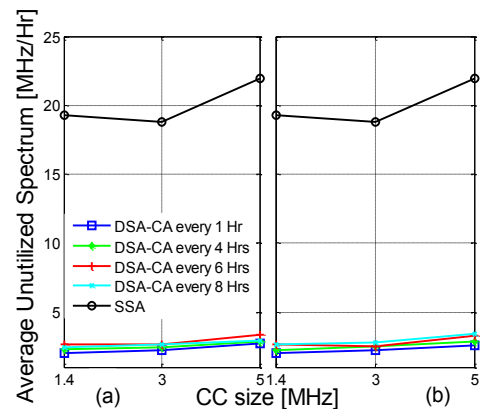

Fig 1. Average unutilized spectrum in case of (a) scenario 1 and (b) scenario 2 .

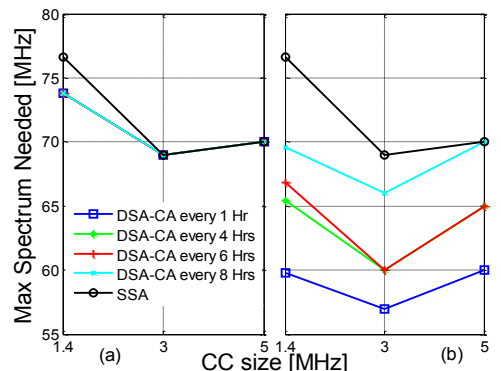

Fig. 2. Maximum Spectrum Needed in case of (a) scenario 1 and (b) scenario 2 .

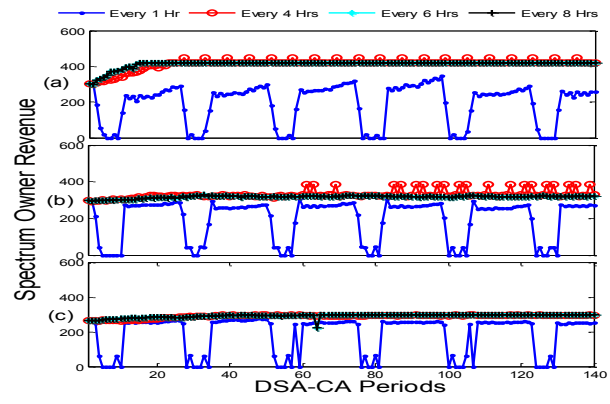

Fig. 3. Revenue of spectrum owner in case of (a) $\mathrm{CC}=$ 1.4 $\mathrm{MHz}$, (b) $\mathrm{CC}=3 \mathrm{MHz}$ and (c) $\mathrm{CC}=5 \mathrm{MHz}$.

\section{CONCLUSIONS}

In this paper, we have presented a realistic DSA-CA framework, feasible DSA for LTE-Advanced networks based on the capabilities of Carrier aggregation. We also have presented an accurate model to estimate the cell's traffic, taking into account the various LTE-Advanced overheads, to estimate the bandwidth needed. This framework presented the bounded knapsack problem as a spectrum auction problem to satisfy the POs demand while maximizing SO's revenue. System level simulations demonstrated a significantly improved performance compared to static spectrum access with more than $20 \%$ reduction in the required spectrum and more than $80 \%$ reduction in the average unutilized spectrum.

\section{REFERENCES}

[1] T. Wenyuan and J. Rahul "Hierarchical auction mechanisms for network Resource allocation," IEEE Journal on Selected Areas in Communications, vol.30, no.11, pp.2117,2125, December 2012.

[2] P. Lin, X. Feng, and Q. Zhang, "Flexauc: Serving Dynamic Demands in Spectrum Trading Markets with Flexible Auction," to appear in IEEE INFOCOM, 2014.

[3] V. Rodriguez, K. Moessner, and R. Tafazolli, "Auction driven dynamic spectrum allocation: optimal bidding, pricing and service priorities for multi-rate, multi-class CDMA," in Proc. of IEEE PIMRC 2005, Sept. 2005.

[4] L. Peng, J. Jia, Q. Zhang, and M. Hamdi, "Dynamic spectrum sharing with multiple primary and secondary users," IEEE Transactions on Vehicular Technology, vol.60, no.4, pp.1756,1765, May 2011.

[5] S. Gandhi, C. Buragohain, L. Cao, H. Zheng, and S. Suri, "A general framework for wireless spectrum auctions," Proceedings of IEEE DySPAN 2007, pp.22-33, 17-20 April 2007

[6] A. Gopinathan, Z. Li, and C. Wu, "Strategyproof auctions for balancing social welfare and fairness in secondary spectrum markets," Proceedings IEEE INFOCOM 2011, vol., no., pp.3020,3028, 10-15 April 2011.

[7] S. Shamik and C. Mainak "Designing auction mechanisms for dynamic spectrum access," Mobile Networks and Applications, vol. 13, No. 5, pp.498-515, 2008.

[8] S. Sengupta, M. Chatterjee, and S. Ganguly, "An economic framework for spectrum allocation and service pricing with competitive wireless service providers," Proceedings of IEEE DySPAN 2007, April 2007.
[9] J. D. Naranjo, G. Bauch, A. B. Saleh, I. Viering, and R. Halfmann, "A dynamic spectrum access scheme for an LTE-Advanced HetNet with carrier aggregation," Proceedings of the 9th International ITG Conference on Systems, Communication and Coding (SCC), 21-24 Jan. 2013.

[10] Y. Xiao, C. Yuen, P. D. Francesco, and L. A. DaSilva, "Dynamic spectrum scheduling for carrier aggregation: A game theoretic approach," Proceedings of IEEE International Conference on Communications (ICC), 2013 , vol., no., pp.2672,2676, 9-13 June 2013.

[11] K. I. Pedersen, F. Frederiksen, C. Rosa, H. Nguyen, L. G. U. Garcia, and Y. Wang, "Carrier aggregation for LTE-Advanced: Functionality and performance," IEEE Communications Magazine, vol.49, no.6, pp.89,95, June 2011.

[12] K. Terence, "Generalized knapsack solvers for multi-unit Combinatorial Auctions: Analysis and Application to Computational Resource Allocation," Theories for and Engineering of Distributed Mechanisms and Systems, pp. 73-86. Springer Berlin Heidelberg, 2005.

[13] S. Shamik and C. Mainak "Designing auction mechanisms for dynamic spectrum access," Mobile Networks and Applications, vol. 13, No. 5, pp.498-515, 2008.

[14] K. Qorany, K. Elsayed, A. Khattab, and F. Digham, " Dynamic Spectrum Access for Primary Operators Via Carrier Aggregation in LTE-Advanced Environments," Technical Report, Cairo University, July $2014 . \quad$ Avialble at: http://eece.cu.edu.eg/ akhattab/files/DSA_CA_Technical_Report.pdf.

[15] F. Digham, K. Elsayed, A. Khattab, and K. Qorany, "Estimation Of Spectrum Demand In An LTL-Advanced Environment," IEEE GLOBECOM Industry (IF\&E) Program, December 2014.

[16] G. Auer, V. Giannini, C. Desset, I. Godor, P. Skillermark, M. Olsson, and et al. "How much energy is needed to run a wireless network?" IEEE Wireless Communications, vol.18, no.5, pp.40-49, October 2011.

[17] E. Dahlman, S. Parkvall, and J. Sköld, "4G LTE/LTE-Aavanced for mobilr broadband," 1st ed., Academic Press, 2011.

[18] A. Larmo, M. Lindstrom, M. Meyer, G. Pelletier, J. Torsner, and H. Wiemann, "The LTE link-layer design," IEEE Communications Magazine, vol.47, no.4, pp.52-59, April 2009.

[19] M. T. Kawser, N. I. B. Hamid, Md. N. Hasan, M. S. Alam, and M. M. Rahman, "Downlink SNR to CQI mapping for different multiple antenna techniques in LTE," International Journal of Information and Electronics Engineering, vol. 2, no. 5, September 2012.

[20] Evolved Universal Terrestrial Radio Access (E-UTRA); Physical downlink shared channel related procedures, 3GPP Technical Specification TS36.213, Rev.10.2.0, Jun 2011. 